\title{
LGAD and 3D as Timing Detectors
}

\section{Sofía Otero Ugobono* on behalf of the RD50 Collaboration.}

Instituto de Microelectrónica de Barcelona (IMB-CNM-CSIC)

E-mail: sofia.otero.ugobonodcsic.es

In view of the High Luminosity upgrade of the CERN Large Hadron Collider (HL-LHC), radiation tolerant silicon sensors are being developed in the framework of ATLAS, CMS, RD50 and other sensor R\&D projects. The HL-LHC beam parameters and hardware configuration should enable the collider to reach a peak instantaneous luminosity of $5 \times 10^{34} \mathrm{~cm}^{-2} \mathrm{~s}^{-1}$, and an integrated luminosity of $250 \mathrm{fb}^{-1} /$ year with the goal of $3000 \mathrm{fb}^{-1}$ after about 12 years of operation. This will imply a factor 5 increase in instantaneous luminosity, and 10 in integrated luminosity with respect to the LHC. This increase in luminosity will also imply a factor 4 rise in the expected pile-up with respect to that observed during Run 2 of the LHC in ATLAS and CMS. Lastly, at the end of the operation period, radiation levels are expected to reach values above $1.6 \times 10^{16}$ fast hadrons $/ \mathrm{cm}^{2}$ at the innermost detectors.

To cope with the increase in pile-up, silicon sensors with timing capabilities of the order of $\sim 30 \mathrm{ps}$ are being developed. Given the expected radiation levels, the radiation-tolerance of these devices is of the utmost importance. In order to tackle these issues, one line of research investigates the possibility of producing radiation tolerant silicon sensors with intrinsic charge gain: Low Gain Avalanche Detectors (LGADs). The aim is to improve the signal height after irradiation as well as the timing capabilities of silicon sensors. Another approach is the use of 3D sensors. The implementation of 3D devices would resolve some of the issues arising from using LGADs such as gain-loss, radiation hardness at fluences beyond $10^{15} \mathrm{~cm}^{-2}$, or a reduced fill factor.

The aim of this paper is to give an overview of both technologies, their performance, and their current development status for timing applications.

The 28th International Workshop on Vertex Detectors - Vertex2019

13-18 October, 2019

Lopud, Croatia

${ }^{*}$ Speaker. 


\section{Introduction}

In view of the High Luminosity upgrade of the CERN Large Hadron Collider (HL-LHC), radiation tolerant silicon sensors are being developed in the framework of the RD50 Collaboration. The HL-LHC envisages a factor 5 increase in instantaneous luminosity and 10 in integrated luminosity with respect to the LHC. The aim is to reach a peak instantaneous luminosity of $5 \times 10^{34} \mathrm{~cm}^{-2} \mathrm{~s}^{-1}$ and an integrated luminosity of $250 \mathrm{fb}^{-1}$ /year, with the goal of $3000 \mathrm{fb}^{-1}$ after about 12 years of operation. Furthermore, the equipment is being designed with a $50 \%$ margin with respect to the wanted peak luminosity: $7-7.5 \times 10^{34} \mathrm{~cm}^{-2} \mathrm{~s}^{-1}$. This pushes the integrated luminosity to $300-350 \mathrm{fb}^{-1}$ year, with the goal of $4000 \mathrm{fb}^{-1}$ after the full operation period [1]. Furthermore, at the end of operation the innermost detectors will have been exposed to radiation levels above $1.6 \times 10^{16}$ fast hadrons $/ \mathrm{cm}^{2}$ [2]. Concurrently, the expected pile-up (the number of interactions per bunch crossing) will be a factor 4 higher than that observed during Run 2 of the LHC in ATLAS and CMS [1, 3, 4]. Whilst ATLAS and CMS had a pile-up of $\sim 50$ during Run 2 [3, 4], at the HL-LHC their pile-up is expected to be between 140 and $200[4,5]$. Since most interactions during Run 2 occurred in clearly distinguishable locations (see Figure 1a), primary vertices ( $p p$ collision) could be reconstructed with a $\sim 99 \%$ efficiency using spatial information only (3D tracking) [6]. In contrast, maintaining the same spatial resolution, at the HL-LHC around 10-15\% of vertices would be in fact overlapped interactions, i.e. vertices separated in space by a distance smaller than the spatial resolution of the detector (see Figure 1b) [7]. To cope with the increase in pile-up, avoid the loss of primary vertices, preserve the event-reconstruction, and exploit the full potential of the luminosity capability of the HL-LHC, timing information must be included (4D tracking) [7].

\section{4D Tracking}

The expected time spread of the beam spot in the HL-LHC when two bunches overlap entirely (maximising the pile-up density) is of $180-200$ ps. If one were to slice up the beam spot in fractions of 30-40 ps, then the pile-up levels in each slice would be of the order of those of the LHC Phase I. Instead of a 10-15\% of primary vertex overlap, in each slice there would only be a $1 \%$ overlap. This means that the LHC Phase-I quality of event reconstruction could be attained using the same spatial resolution as in Phase I by adding a time resolution per track of at least $30-40$ ps [8].

To properly reconstruct events, the timing information can be obtained and applied to the reconstruction process at different stages. One possibility is to assign a timestamp to each hit on the tracking system, i.e. to every single point of a track, see Figure 2. Developing such a tracking system is a major challenge from the point of view of the detectors themselves and the read-out electronics $[9,10]$. Another option is to directly time-tag tracks instead of individual hits (see Figure 3), which is less demanding on the read-out and processing electronics. In this case, the timing is carried out on dedicated timing layers either inside or outside the tracker volume, requiring minimal changes to the main tracker hardware[7]. 


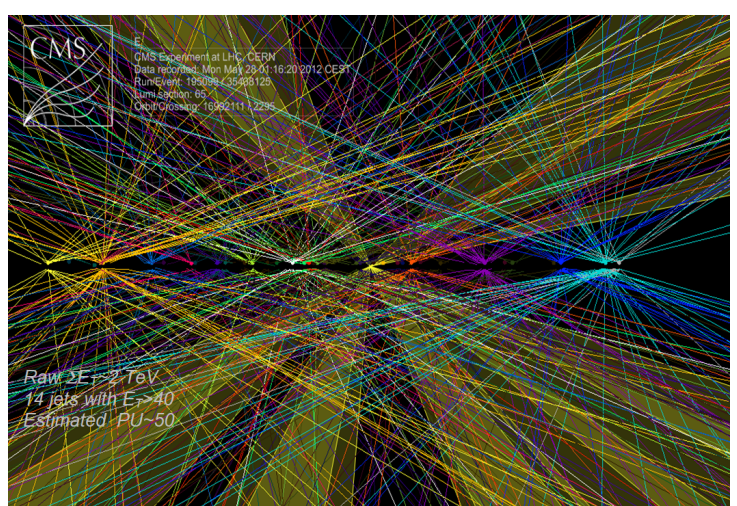

(a)

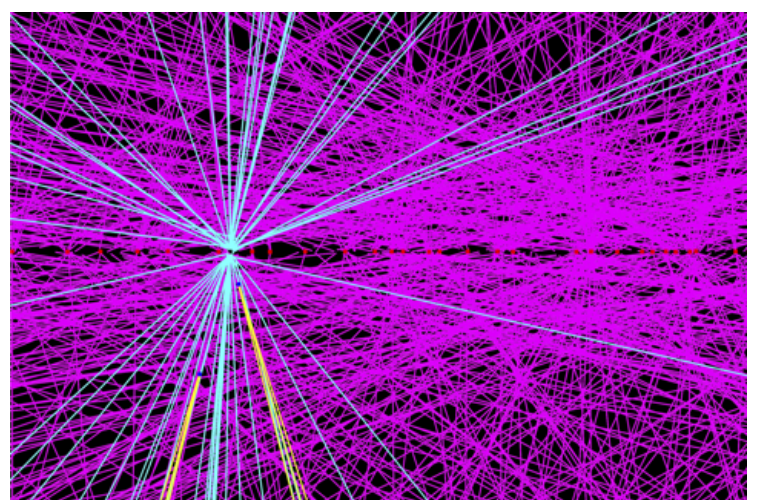

(b)

Figure 1: (a) Illustration of a real CMS event with a pile-up of $\sim 50$. From [11]. (b) Simulation of an ATLAS collision at the HL-LHC with an average pile-up of 200. From [12].
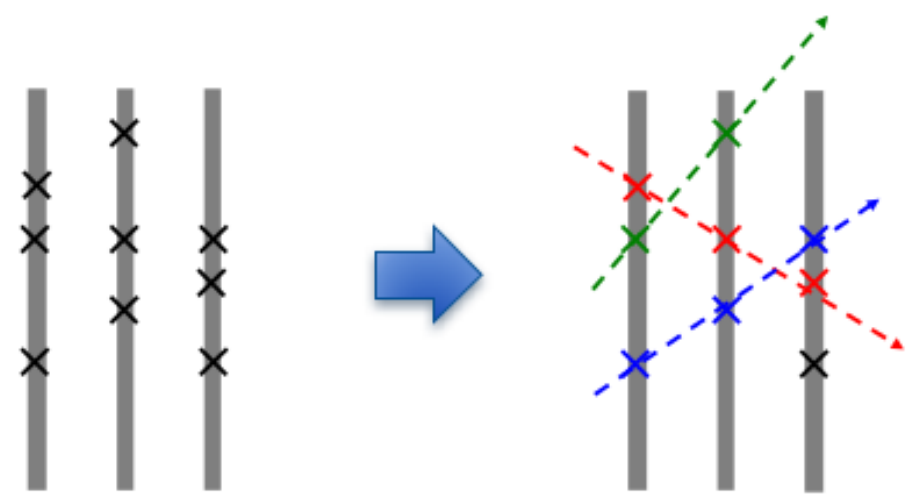

Figure 2: With spatial information only it would not be possible to reconstruct the tracks from this pattern of hits (left). Including precise timing information enables the reconstruction of tracks by associating timecompatible hits (right). From [9].
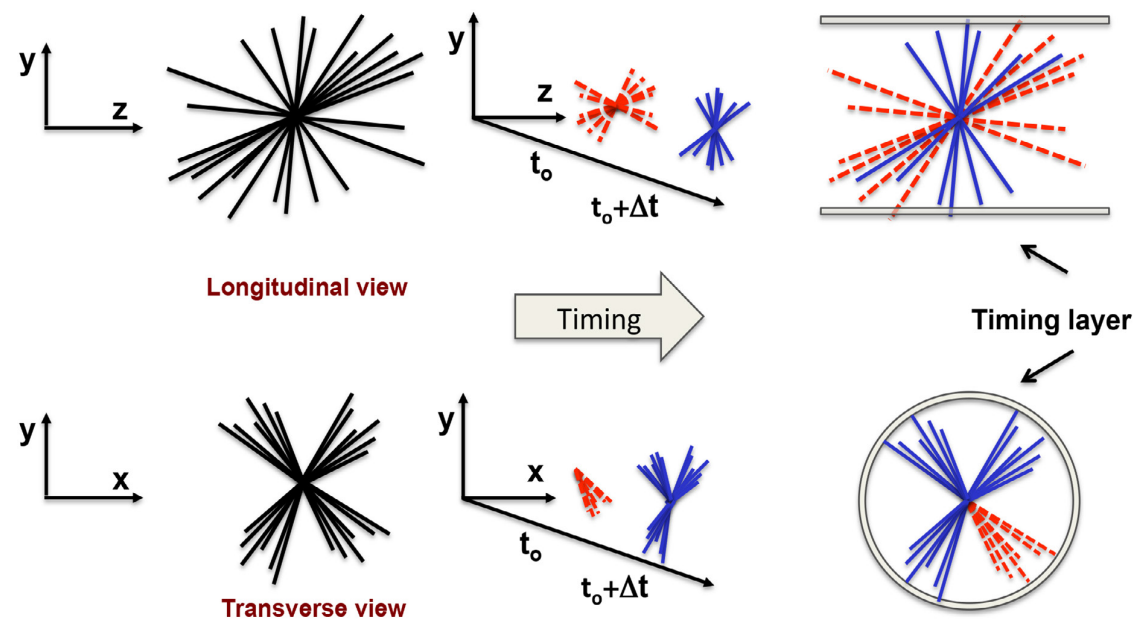

Figure 3: Simplified graphical explanation of how time-tagging tracks by means of a timing layer can be used to distinguish two vertices that overlap in space. From [7]. 


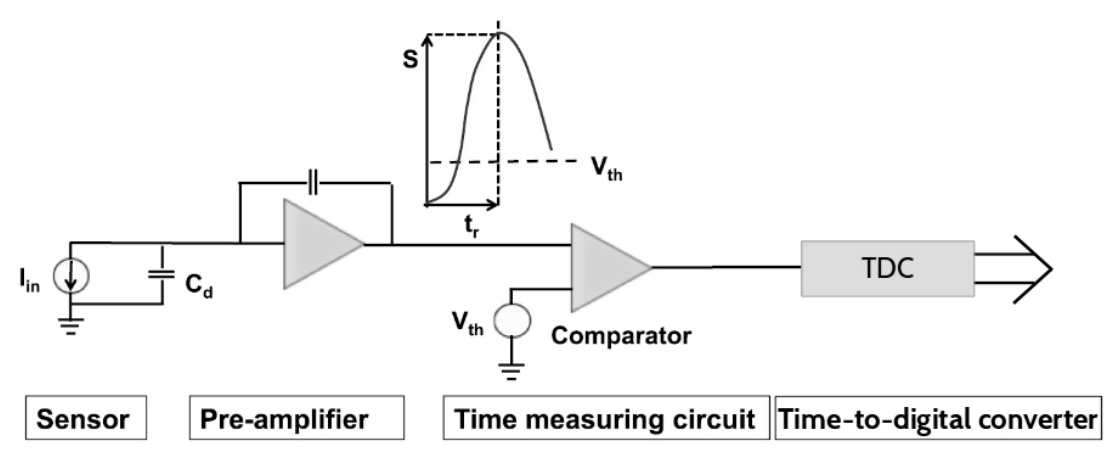

Figure 4: Schematic of the main components of a time-tagging detector. The sensor is represented by a capacitor with a current source in parallel. Modified from [13].

\subsection{Time resolution}

Regardless of the method used for 4D tracking, in order to establish the required properties of timing sensors, it is necessary to know which parameters affect the time resolution. Basically, each step in the read-out process and any effect that changes the shape of the signal has a different impact in the time resolution. Figure 4 shows a schematic of the main components of a time-tagging detector, including the read-out electronics. The silicon sensor is represented by a capacitor with a current source in parallel. The read-out is performed through a preamplifier and its output is compared to a threshold $\left(\mathrm{V}_{\mathrm{th}}\right)$ to determine the time of arrival $\left(\mathrm{t}_{0}\right)$. The latter is digitised by means of a time-to-digital converter (TDC). The expression for the time resolution, seen in Equation 2.1, takes into consideration the contributions of each of the previously described steps. In the following sections each term in Equation 2.1 is discussed.

$$
\sigma_{t}^{2}=\sigma_{\text {Time walk }}^{2}+\sigma_{\text {Landau noise }}^{2}+\sigma_{\text {Distortion }}^{2}+\sigma_{\text {Jitter }}^{2}+\sigma_{\text {TDC }}^{2}
$$

\subsubsection{Time walk}

The variations in time of arrival due to different signal amplitudes is called time walk [14] (illustrated in Figure 5a) and it is defined as $\sigma_{\text {Time walk }} \propto\left[\frac{N \sigma_{n}}{\mathrm{dV} / \mathrm{dt}}\right]_{R M S}$. Here the threshold voltage is expressed in multiples of the system noise $\left(N \sigma_{n}\right)$. The denominator represents the slew rate $(\mathrm{dV} / \mathrm{dt})$, i.e. the change in voltage or current per unit time [7]. Time walk can be reduced by minimising the noise, maximising the slew rate, and by setting the threshold to the lowest possible level. Also, time walk can be mitigated by using amplitude compensating circuitry. Further details can be found in [7].

\subsubsection{Landau noise}

When a given MIP goes through a Si sensor, its energy deposition per unit length is not uniform. These fluctuations, referred to as Landau noise, alter the shape of the induced signal in devices with localised multiplication of charge. Given its intrinsic nature, Landau noise can be minimised, but not eliminated. In fact, it sets a physical limit to the precision of a silicon sensor. Nevertheless, Landau noise can be minimised by setting the threshold as low as possible, and by using thinner detectors [17]. 


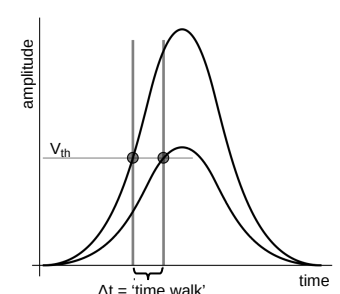

(a)

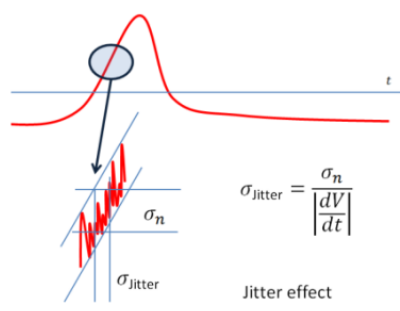

(b)

Figure 5: Graphical representation of (a) time walk: the variation in time of arrival due to differences in signal amplitude (modified from [15]); (b) the jitter effect (modified from [16]).

\subsubsection{Signal distortion: drift velocity and weighting field}

Since the signal induced by charge carriers is proportional to the drift velocity and the weighting field, any inhomogeneities in these two parameters will cause a dependence of the timing resolution on the hit position. The simplest solution to avoid this is to saturate the drift velocity in the bulk and to optimise the sensor geometry so that the weighting field is fairly constant $[7,17]$.

\subsubsection{Jitter}

Jitter is a consequence of the variations in time of arrival due to signal and electronic noise. By a simple geometrical projection, shown in Figure 5b, the jitter can be determined using the equation $\sigma_{\mathrm{Jitter}}=\frac{\sigma_{n}}{\mathrm{dV} / \mathrm{dt}}$. The jitter can be minimised by using timing detectors with low intrinsic noise and very fast slew rates, as well as low-noise read-out electronics [7].

\subsubsection{TDC effects}

The contribution of time-to-digital converters (TDCs) to the time resolution is minimal. It is associated to the time bin width $(\Delta T)$ used to digitise the time of arrival. Current TDCs have sub-picosecond time resolution, rendering their contribution to the timing resolution $(\Delta T / \sqrt{12})$ negligible compared to the other terms $[7,18]$.

\subsection{Detector requirements for timing}

Based on the contributing factors described, it can be concluded that timing detectors must have very fast slew rates and low intrinsic noise to minimise the jitter and the time walk. In addition, the use of thin devices is recommended, particularly so in devices with intrinsic gain where Landau noise is particularly significant. Finally, low noise read-out electronics are required.

\section{Low Gain Avalanche Detectors}

Low Gain Avalanche Detectors (LGADs) are planar silicon sensors with intrinsic gain [19]. They consist of a high-resistivity $(\sim 12 \mathrm{k} \Omega)$ p-type bulk with a p++ ohmic contact, an $\mathrm{n}+$ electrode, and a diffused $\mathrm{p}+$ (multiplication) layer just below the $\mathrm{n}+$ electrode, see Figure 6. When an LGAD is reverse biased, a high electric field region is produced in the multiplication layer. If the electric field there is high enough $(>200 \mathrm{kV} / \mathrm{cm})$, any electrons going through this region will undergo 


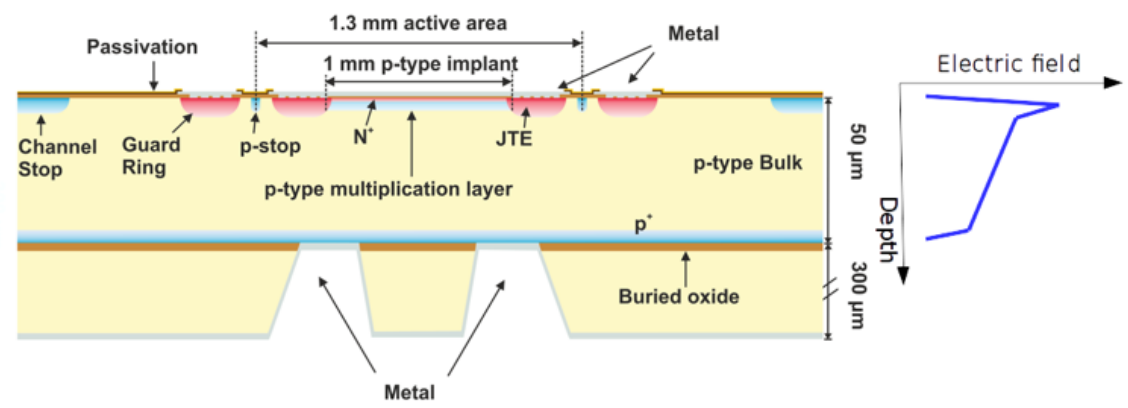

Figure 6: Cross-section schematic of an LGAD. On the right, a depiction of the electric field inside an LGAD is shown.

impact ionisation causing an avalanche of additional charge carriers. The doping concentration of the multiplication layer, the bias voltage applied, and the temperature of operation determine the extent of the multiplication [20,21]. For timing applications the gain must be optimised to allow for an increase in the signal whilst maintaining low noise levels, a low risk of breakdown, and a moderate power consumption even after irradiation [7].

Several production runs of LGADs have been fabricated, first by the Centro Nacional de Microelectrónica (CNM), followed by Hamamatsu Photonics K.K. (HPK), Fondazione Bruno Kessler (FBK), Micron Semiconductor Ltd., Brookhaven National Laboratory, and IHEP-NDL. The development of LGADs is considerably advanced, in fact they have already been installed in the CMS CT-PPS experiment and various projects (ATLAS HGTD, CMS ETL, AFP, and TOTEM) foresee their use in their detectors $[8,22,23]$. There is a varied range of LGAD designs available, from different thicknesses and active areas to different layouts. In particular, thin devices of thicknesses between 35 and $50 \mu \mathrm{m}$ are available. As regards layout, both individual pads and pad arrays can be produced.

\subsection{LGAD performance}

During the past years there has been a continuous effort to conduct radiation-hardness and timing tests LGADs. A brief summary of the most notable results on timing resolution and radiation tolerance are described here, as well as the latest results on fill-factor improvements.

The evolution with fluence of the gain and the time resolution of $50 \mu \mathrm{m}$-thick HPK LGADs with an active area diameter of $1.0 \mathrm{~mm}$ was studied in [24]. The devices were irradiated with neutrons at the Jožef Stefan Institute in Ljubljana, Slovenia. The gain and time resolution were determined by performing ${ }^{90} \mathrm{Sr}$ source measurements at $-20^{\circ} \mathrm{C}$, the results obtained can be seen in Figures $7 \mathrm{a}$ and $7 \mathrm{~b}$. The gain was found to decrease with fluence. This is attributed to acceptor removal: the decrease in effective doping of p-type silicon due to irradiation. Boron atoms are removed from their lattice locations (substitutional state) and get electrically deactivated by forming complexes with other elements [25]. In the specific case of LGADs, if the effective doping of the multiplication layer decreases, then the gain of the device decreases as well. However, it was found that the gain could be recovered by simply increasing the bias voltage applied. Having said that, at fluences above $10^{15} \mathrm{neq} / \mathrm{cm}^{2}$, because of acceptor removal, the gain layer is not longer active. Hence, the gain observed is caused by the multiplication of charge carriers in the bulk of the sen- 


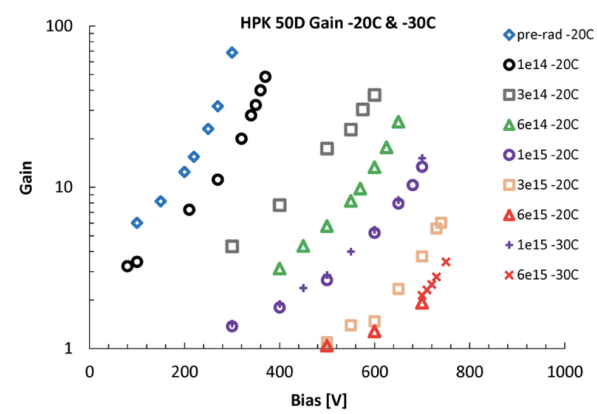

(a)

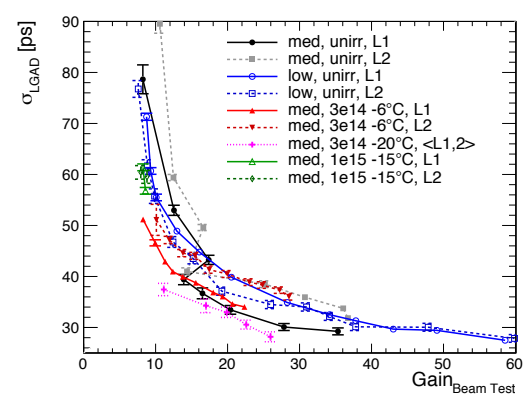

(c)

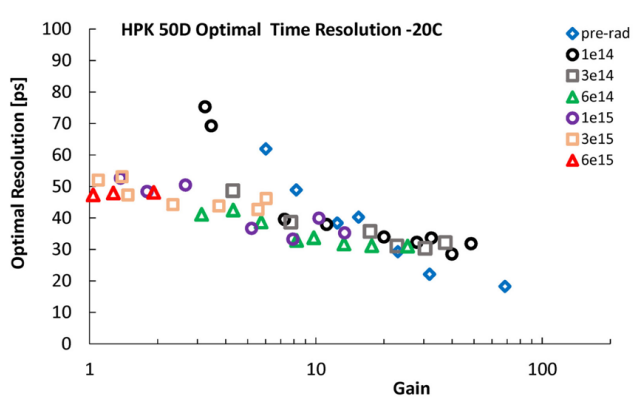

(b)

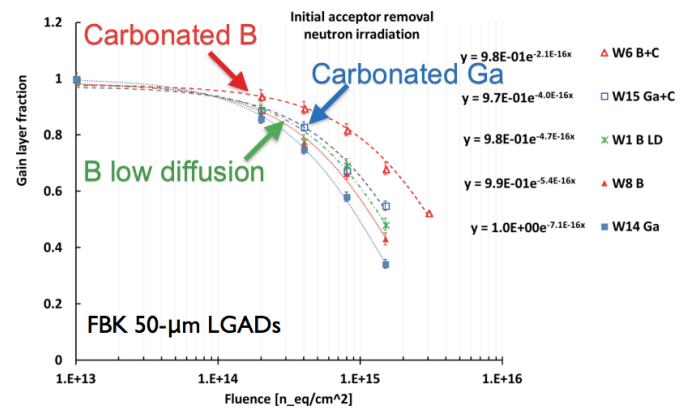

(d)

Figure 7: (a) Gain as a function of bias and (b) time resolution as a function of gain for $50 \mu \mathrm{m}$-thick HPK LGADs [24]. (c) Time resolution as a function of gain for $45 \mu \mathrm{m}$-thick CNM LGADs [26]. (d) Active fraction of the gain layer as a function of fluence [27]. All irradiations were done with neutrons.

sor, as the electric field in the bulk is high enough to cause impact ionisation. Regarding the time resolution $\left(\sigma_{t}\right)$, up to a fluence of $3 \times 10^{14} \mathrm{neq} / \mathrm{cm}^{2}$ it remains below or around $30 \mathrm{ps}$. On the other hand, at $6 \times 10^{15} \mathrm{neq} / \mathrm{cm}^{2}$, the highest fluence evaluated, $\sigma_{t}$ reached $50 \mathrm{ps}$.

A similar investigation was carried out on $45 \mu \mathrm{m}$-thick CNM LGADs with a $1.3 \times 1.3-\mathrm{mm}^{2}$ active area and can be found in [26]. The devices were also irradiated with neutrons at the Jožef Stefan Institute in Ljubljana, Slovenia. The timing studies of these sensors were done during the AFP beam tests of 2016, using the H6B beam line with 120-GeV pions at the CERN-SPS North Area. The time resolution as a function of gain for all tested fluences can be seen in Figure 7c. Up to $3 \times 10^{14} \mathrm{neq} / \mathrm{cm}^{2}$ the time resolution remains below $30 \mathrm{ps}$ at $-20^{\circ} \mathrm{C}$. The sample irradiated to $10^{15} \mathrm{neq} / \mathrm{cm}^{2}$ was measured at $-15^{\circ} \mathrm{C}$, achieving a minimum time resolution of $57 \mathrm{ps}$.

The results obtained with HPK and CNM sensors are fairly similar, showing that at fluences of the order of $10^{15} \mathrm{neq} / \mathrm{cm}^{2}$ the time resolution is above the sought-after resolution of $30 \mathrm{ps}$. Having said that, the aforementioned target resolution is per track, not per hit. One way of improving the overall timing performance of $4 \mathrm{D}$ tracking systems is by using multiple overlapping sensor layers. Under these conditions, the time resolution per track would be given by the following equation:

$$
\sigma_{t}^{\text {per track }}=\frac{\sigma_{t}^{\text {per hit }}}{\sqrt{\text { Number of hits }}} .
$$

Hence, by placing the detector modules in each layer in such a way that the overlap between sensors increases the number of hits per track, the overall timing resolution can be improved even 
at very high fluences. For instance, the HGTD design contemplates the use of four layers where the overlap between sensors (LGADs) depends on their radial position $R$ with respect to the beam axis. For modules in $R>320 \mathrm{~mm}$ an overlap of $20 \%$ ensures an average of two hits per passing particle. The innermost modules $(R<320 \mathrm{~mm})$, exposed to the highest radiation doses, will have an $80 \%$ overlap, resulting, on average, in at least three hits [28]. Consequently, with the LGADs tested in the studies discussed in this section, the time resolution per track would be around the wanted $30 \mathrm{ps}$ even at the highest fluences analysed. For example, assuming the expected average of 3 hits in the HGTD, the HPK LGADs irradiated up to $6 \times 10^{15} \mathrm{neq} / \mathrm{cm}^{2}\left(\sigma_{t}=50 \mathrm{ps}\right.$ ) would result (following Equation 3.1) in a time resolution per track of $\sim 28.9$ ps.

Improvements in the performance of LGADs are not limited to the minimisation of the time resolution. Another important aspect is their radiation hardness. Various studies focus on this issue, in particular a comprehensive study of the radiation hardness of different doping profiles of $50 \mu \mathrm{m}$-thick FBK LGADs is reported in [27]. The experimental results showed that gallium-doped LGADs, which were expected to be less prone to acceptor removal, were in fact more heavily affected by it than those doped with boron. Meanwhile, the carbon enrichment of the gain layer, either gallium- or boron-doped, reduced the impact of acceptor removal. Moreover, thinner and more doped gain layers are less vulnerable to acceptor removal. This was determined by testing the radiation hardness of low-diffusion boron gain layers in comparison to standard boron implants. All of these results are summarised in Figure 7d, where the remaining active fraction of the gain layer is shown as a function of the fluence. The more radiation tolerant the LGAD, i.e. the less susceptible to acceptor removal, the larger the remaining active fraction.

A different line of action for improving the radiation hardness of LGADs is to cleverly design the 4D tracking systems. A clear example is the proposed designed for the HGTD where the innermost modules will be replaced after half of the lifetime of the detector [29].

Finally, there is the issue of the fill factor. The simplest strategy to tackle this problem is the use of LGAD pad arrays instead of individual pads. Still, there are dead regions between pads. In part, these are caused by the geometry of the pads themselves [29]. Although the biggest issue are the 40-100 $\mu \mathrm{m}$-wide no-gain regions due to junction termination structures [30]. Therefore, the next logical step is to produce segmented LGADs. The most straightforward approach is to segment a standard LGAD, as shown in Figure 8a, which implies that the multiplication layer is segmented. However, this design results in a spatially inhomogeneous gain, meaning that the multiplication of charge depends on the particle hit position. Thus, the time and position resolutions are degraded in the case of inter-strip hits, which does not solve the fill-factor problem. To achieve a uniform gain distribution, the multiplication layer must be left intact and the segmentation done on the pside, resulting in a p-in-p device, see Figure 8b. The timing capabilities of an unirradiated 285 $\mu \mathrm{m}$-thick iLGAD produced by CNM were analysed in [31] and the results are promising. The timing resolution of the device was measured using a picosecond pulsed infrared laser, obtaining a resolution of $\sim 20$ ps at $20^{\circ} \mathrm{C}$. Given that the measurements were done with a laser, Landau flactuations and their contributions to the time resolution are not evaluated. The timing resolution of that same device for MIPs will be worse. In order to further improve the time resolution for MIPs, thinner iLGADs are under development. 


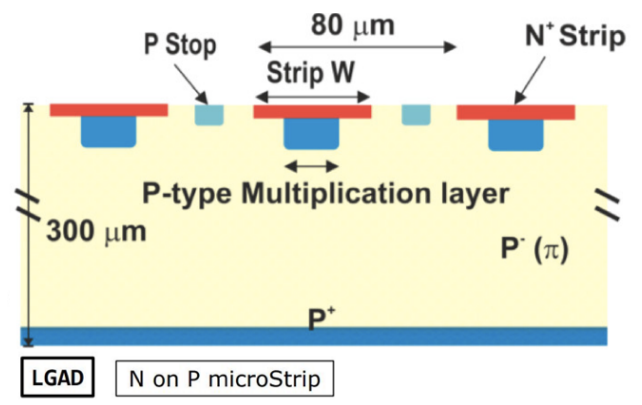

(a)

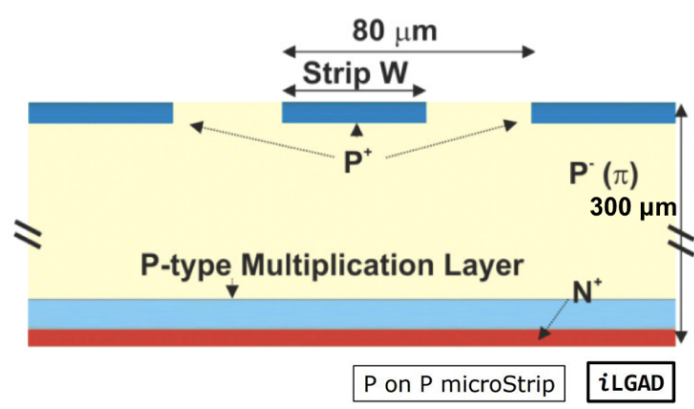

(b)

Figure 8: Cross-section schematic of (a) a segmented LGAD, (b) an inverted LGAD (iLGAD) [31].

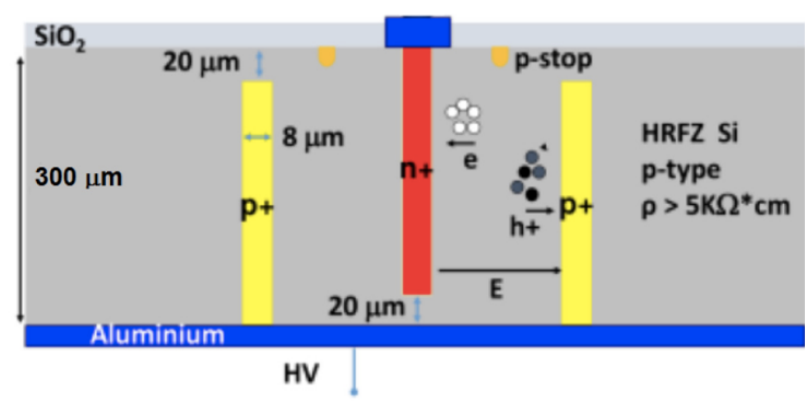

(a)

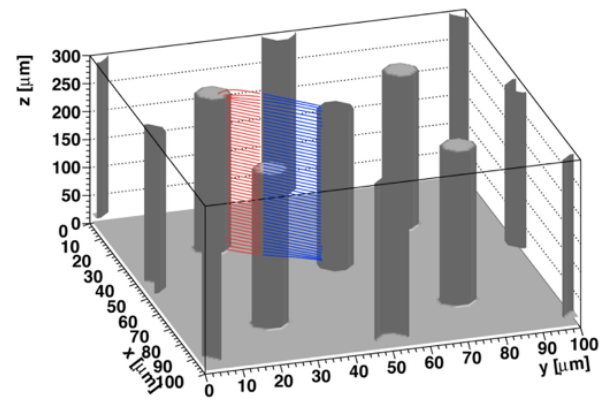

(b)

Figure 9: (a) Cross-section schematic of a double-sided 3D sensor. (b) Schematic showing the drift paths of MIP-induced electrons (blue) and holes (red). Electrons move towards a junction column and holes to an ohmic column [30].

\section{3D sensors}

These devices use columnar electrodes which penetrate through the bulk of the sensor, as seen in Figure 9a [32]. As a result, the drift distance is decoupled from the sensor thickness, clearly depicted in Figure $9 \mathrm{~b}$ where the drift path of $\mathrm{e}^{-} / \mathrm{h}^{+}$pairs formed by a MIP are shown. Consequently, the same large signals expected from thick planar detectors can be obtained whilst requiring lower depletion voltages and reducing the power dissipation. In addition, the charge collection is faster and trapping due to defects is reduced, which lead to 3D sensors being more radiation tolerant. In fact, 3D devices with small cell sizes can work up to fluences of the order of $10^{16} \mathrm{n}_{\mathrm{eq}} / \mathrm{cm}^{2}$ [33, 34]. Another benefit of 3D sensors is their close to $100 \%$ fill factor, a great advantage when compared to pad sensors [30]. All of these properties, specially so the fill factor, the radiation hardness, and the short drift times make 3D sensors a promising technology for timing applications in high radiation environments.

The timing capabilities of 3D sensors are currently under study and so far the results have been promising. In [30], the timing capabilities of a $300 \mu$ m-thick CNM 3D sensor with a p-type bulk and a $50 \times 50 \mu \mathrm{m}^{2}$ cell size were both measured and simulated. A cross section of the device under test can be seen in Figure 9a. Each square cell consists of four $p^{+}$ohmic columns at each corner of the cell and an $n^{+}$junction electrode at the centre. Timing measurements were carried out in [30] 


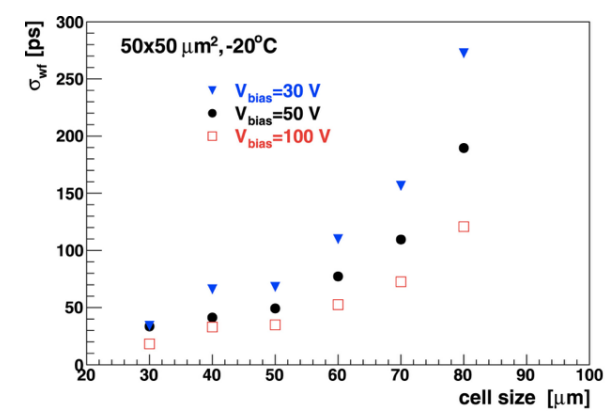

(a)

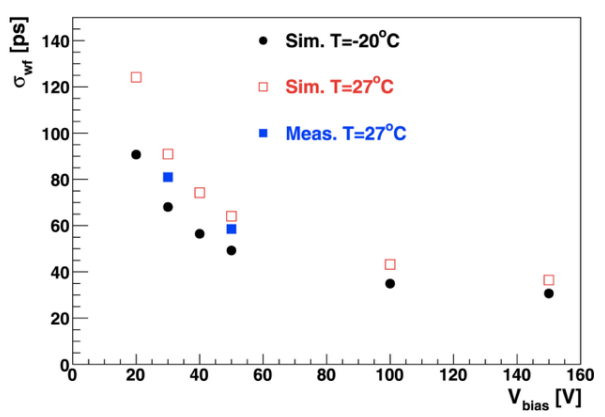

(b)

Figure 10: Simulation results: $\sigma_{\mathrm{wf}}$ as a function of (a) square-cell side length for different bias voltages at $-20^{\circ} \mathrm{C}$, (b) bias voltage for a $50 \times 50-\mu \mathrm{m}^{2}$ cell at $-20^{\circ} \mathrm{C}$ and $27^{\circ} \mathrm{C}$ (two experimentally obtained data points are included) [30].

at $20^{\circ} \mathrm{C}$ using a ${ }^{90} \mathrm{Sr}$ source and applying a bias voltage of $50 \mathrm{~V}$. Under these conditions, the time resolution was of $75 \mathrm{ps}$. The main contributions to the time resolution were found to be the jitter and the time walk. The latter is dominated by the hit position inside the cell. Since the weighting field in 3D sensors is not uniform, the drift velocity is not constant and the signal shape is affected by the drift path of the charge carriers. Simulations were carried out to determine how to minimise the hit-position contribution to the time resolution $\left(\sigma_{\mathrm{wf}}\right)$, see Figure 10. It was determined that $\sigma_{\mathrm{wf}}$ can be minimised by employing devices with smaller cells, increasing the bias voltage, and reducing the temperature, which increases the drift velocity. Furthermore, it was found that the hit-position contribution for perpendicular tracks is higher than for inclined ( $5^{\circ}$ angle) tracks, $54 \mathrm{ps}$ and $51 \mathrm{ps}$ respectively. Lastly, if instead of separately reading out each cell, multiple cells are read out together, the timing resolution is further improved, particularly for angled tracks. At $-20^{\circ} \mathrm{C}$ and applying a $50 \mathrm{~V}$ bias voltage, simulations predict a $\sigma_{\mathrm{wf}}$ of $46 \mathrm{ps}$ for perpendicular tracks with individual cell read-out and of $20 \mathrm{ps}$ for $5^{\circ}$ tracks in multi-cell read-out mode.

\section{Conclusions}

In view of the upcoming High Luminosity upgrade of the Large Hadron Collider, the addition of timing information for track reconstruction is vital. This paper summarises some of the latest developments achieved within the framework of the RD50 Collaboration on timing sensors.

The development of LGADs is considerably advanced, with various projects foreseeing their use. Thin LGADs $(35-50 \mu \mathrm{m})$ are readily available, with time resolutions below $30 \mathrm{ps}$ at $-20^{\circ} \mathrm{C}$ up to fluences of $3 \times 10^{14} \mathrm{neq} / \mathrm{cm}^{2}$. Also, the overall time resolution per track can be improved by using multiple sensor layers. Still, there is on-going work to further improve the radiation hardness of LGADs and solve the current fill-factor issues by producing thin iLGADs.

3D sensors are a most promising technology for timing applications in harsh environments due to their short drift times, $\sim 100 \%$ fill-factor, and high radiation tolerance (up to $10^{16} \mathrm{neq} / \mathrm{cm}^{2}$ ). Their time resolution has been found to be dominated by the hit-position contribution, which simulations predict can be minimised by using smaller cells, multiple-cell read-out, and lower operation temperatures. The results are promising for timing applications. Still, further studies are needed. 


\section{References}

[1] G. Apollinari et al., High-Luminosity Large Hadron Collider (HL-LHC): Technical Design Report V. 0.1, ser. CERN Yellow Reports: Monographs. Geneva: CERN, 2017. [Online]. Available: https://cds.cern.ch/record/2284929

[2] F. Gianotti et al., "Physics potential and experimental challenges of the LHC luminosity upgrade," Eur. Phys. J. C, vol. 39, no. 3, pp. 293-333, 2005. [Online]. Available: http://dx.doi.org/10.1140/epjc/s2004-02061-6

[3] F. Pastore; on behalf of the ATLAS Collaboration, "ATLAS Run-2 status and performance," Nucl. Part. Phys. Proc., vol. 270-272, pp. 3 - 7, 2016, 18th Montpellier International Conference on Quantum Chromodynamics (QCD 15). [Online]. Available: http://www.sciencedirect.com/science/article/pii/S2405601416000031

[4] D. Contardo et al., "Technical Proposal for the Phase-II Upgrade of the CMS Detector," CERN, Geneva, Tech. Rep. CERN-LHCC-2015-010. LHCC-P-008. CMS-TDR-15-02, Jun 2015. [Online]. Available: https://cds.cern.ch/record/2020886

[5] ATLAS Collaboration, “ATLAS Phase-II Upgrade Scoping Document," CERN, Geneva, Tech. Rep. CERN-LHCC-2015-020. LHCC-G-166, Sep 2015. [Online]. Available: https://cds.cern.ch/record/2055248

[6] K. Grimm et al., "Primary vertex reconstruction at the ATLAS experiment," CERN, Geneva, Tech. Rep. ATL-SOFT-PROC-2017-051. 4, Feb 2017. [Online]. Available: https://cds.cern.ch/record/2253428

[7] H. F.-W. Sadrozinski, A. Seiden, and N. Cartiglia, "4D tracking with ultra-fast silicon detectors," Reports on Progress in Physics, vol. 81, no. 2, p. 026101, 2018. [Online]. Available: http://stacks.iop.org/0034-4885/81/i=2/a=026101

[8] CMS Collaboration, "Technical Proposal for a MIP Timing Detector in the CMS Experiment Phase 2 Upgrade,” CERN, Geneva, Tech. Rep. CERN-LHCC-2017-027. LHCC-P-009, Dec 2017. [Online]. Available: https://cds.cern.ch/record/2296612

[9] M. Fiorini et al., "4D fast tracking for experiments at the High Luminosity LHC," in Proceedings of the 25th International Workshop on Vertex Detectors: La Biodola, Isola d'Elba, Italy, September 26-30, 2016 - PoS(Vertex 2016), vol. 287, 2017, p. 067. [Online]. Available: https://pos.sissa.it/287/067/pdf

[10] N. Neri et al., "4D fast tracking for experiments at high luminosity LHC," Journal of Instrumentation, vol. 11, no. 11, p. C11040, 2016. [Online]. Available: http://stacks.iop.org/1748-0221/11/i=11/a=C11040

[11] C. Tully, "Hadron Collider Environment," presented at the 40th SLAC Summer Institute, SLAC National Accelerator Laboratory, California, USA, 2012. [Online]. Available: https://indico.cern.ch/event/200595/contributions/381689/

[12] ATLAS Collaboration, "Simulated HL-LHC collision event in the ATLAS detector," May 2019, general Photo. [Online]. Available: http://cds.cern.ch/record/2674770

[13] N. Cartiglia et al., "Performance of ultra-fast silicon detectors," Journal of Instrumentation, vol. 9, no. 02, p. C02001, 2014. [Online]. Available: http://stacks.iop.org/1748-0221/9/i=02/a=C02001

[14] H. Spieler, Semiconductor Detector Systems. Oxford, Great Britain: Oxford University Press, 2005.

[15] [Online]. Available: https://commons.wikimedia.org/wiki/File:Constant_fraction_1.svg 
[16] N. Cartiglia et al., "First results on the timing resolution properties of LGAD," presented at the 25th RD50 Workshop, CERN, Geneva, Switzerland, 2014.

[17] N. Cartiglia et al., "Tracking in 4 dimensions," Nucl. Instrum. Meth. A, vol. 845, pp. 47 - 51, 2017, proceedings of the Vienna Conference on Instrumentation 2016. [Online]. Available: http://www.sciencedirect.com/science/article/pii/S0168900216304715

[18] A. Rivetti, "Fast front-end electronics for semiconductor tracking detectors: Trends and perspectives," Nucl. Instrum. Meth. A, vol. 765, pp. 202 - 208, 2014, Proceedings of the 9th International "Hiroshima" Symposium on Development and Application of Semiconductor Tracking Detectors. [Online]. Available: http://www.sciencedirect.com/science/article/pii/S016890021400374X

[19] G. Pellegrini et al., "Technology developments and first measurements of Low Gain Avalanche Detectors (LGAD) for high energy physics applications," Nucl. Instrum. Meth. A, vol. 765, pp. 12 16, 2014, Proceedings of the 9th International "Hiroshima" Symposium on Development and Application of Semiconductor Tracking Detectors. [Online]. Available: http://www.sciencedirect.com/science/article/pii/S0168900214007128

[20] D. R. Decker and C. N. Dunn, "Temperature dependence of carrier ionization rates and saturated velocities in silicon,” Journal of Electronic Materials, vol. 4, no. 3, pp. 527-547, Jun 1975. [Online]. Available: https://doi.org/10.1007/BF02666234

[21] V. Greco et al., "Silicon Devices Optimised for Avalanche Multiplication," in Proceedings of the 23rd International Workshop on Vertex Detectors: Doksy, Czech Republic, September 15-19, 2014 PoS(Vertex 2014), vol. 227, 2015, p. 031. [Online]. Available: http://pos.sissa.it/archive/conferences/227/031/Vertex2014_031.pdf

[22] C. Agapopoulou, "A High-Granularity Timing Detector for the Phase-II upgrade of the ATLAS Detector System,” CERN, Geneva, Tech. Rep. ATL-LARG-PROC-2017-004, Nov 2017. [Online]. Available: http://cds.cern.ch/record/2293126

[23] The CMS and TOTEM Collaborations, "CMS-TOTEM Precision Proton Spectrometer," CERN, Tech. Rep. CERN-LHCC-2014-021. TOTEM-TDR-003. CMS-TDR-13, Sep 2014. [Online]. Available: https://cds.cern.ch/record/1753795

[24] Z. Galloway et al., "Properties of HPK UFSD after neutron irradiation up to $6 \mathrm{e} 15 \mathrm{n} / \mathrm{cm}^{2}$," Nucl. Instrum. Meth. A, vol. 940, pp. 19 - 29, 2019. [Online]. Available: http://www.sciencedirect.com/science/article/pii/S0168900219306278

[25] R. Wunstorf et al., "Investigations of donor and acceptor removal and long term annealing in silicon with different boron/phosphorus ratios," Nucl. Instrum. Meth. A, vol. 377, no. 2, pp. 228 - 233, 1996, proceedings of the Seventh European Symposium on Semiconductor. [Online]. Available: http://www.sciencedirect.com/science/article/pii/0168900296002173

[26] J. Lange et al., "Gain and time resolution of $45 \mu \mathrm{m}$ thin low gain avalanche detectors before and after irradiation up to a fluence of $10^{15} \mathrm{n}_{\mathrm{eq}} / \mathrm{cm}^{2}$," Journal of Instrumentation, vol. 12, no. 05, pp. P05 003-P05 003, may 2017. [Online]. Available: https://doi.org/10.1088\%2F1748-0221\%2F12\%2F05\%2Fp05003

[27] M. Ferrero et al., "Radiation resistant LGAD design," Nucl. Instrum. Meth. A, vol. 919, pp. 16 - 26, 2019. [Online]. Available: http://www.sciencedirect.com/science/article/pii/S0168900218317741

[28] C. Allaire, "A High-Granularity Timing Detector in ATLAS: Performance at the HL-LHC," Nucl. Instrum. Meth. A, vol. 924, pp. 355 - 359, 2019, Proceedings of the 11th International "Hiroshima" Symposium on Development and Application of Semiconductor Tracking Detectors. [Online]. Available: http://www.sciencedirect.com/science/article/pii/S0168900218306284 
[29] A. Collaboration, "Technical Proposal: A High-Granularity Timing Detector for the ATLAS Phase-II Upgrade,” CERN, Geneva, Tech. Rep. CERN-LHCC-2018-023. LHCC-P-012, Jun 2018. [Online]. Available: http://cds.cern.ch/record/2623663

[30] G. Kramberger et al., "Timing performance of small cell 3D silicon detectors," Nucl. Instrum. Meth. A, vol. 934, pp. 26 - 32, 2019. [Online]. Available: http://www.sciencedirect.com/science/article/pii/S0168900219305704

[31] E. Currás et al., "Inverse Low Gain Avalanche Detectors (iLGADs) for precise tracking and timing applications," in 15th Vienna Conference on Instrumentation (VCI2019) Vienna, Austria, February 18-22, 2019, 2019.

[32] G. Pellegrini et al., "3D double sided detector fabrication at IMB-CNM," Nucl. Instrum. Meth. A, vol. 699, pp. 27 - 30, 2013, Proceedings of the 8th International "Hiroshima" Symposium on Development and Application of Semiconductor Tracking Detectors. [Online]. Available: http://www.sciencedirect.com/science/article/pii/S0168900212006092

[33] S. Parker, C. Kenney, and J. Segal, "3D - A proposed new architecture for solid-state radiation detectors," Nucl. Instrum. Meth. A, vol. 395, no. 3, pp. 328 - 343, 1997, proceedings of the Third International Workshop on Semiconductor Pixel Detectors for Particles and X-rays. [Online]. Available: http://www.sciencedirect.com/science/article/pii/S0168900297006943

[34] J. Lange et al., "Radiation hardness of small-pitch 3D pixel sensors up to a fluence of $3 \times 10^{16} \mathrm{n}_{\mathrm{eq}} / \mathrm{cm}^{2}$," Journal of Instrumentation, vol. 13, no. 09, pp. P09 009-P09 009, sep 2018. [Online]. Available: https://doi.org/10.1088\%2F1748-0221\%2F13\%2F09\%2Fp09009 